\title{
Maximizing the minimum completion time on parallel machines
}

\author{
Mohamed Haouari • Mahdi Jemmali
}

Received: 18 October 2006 / Revised: 15 March 2007 / Published online: 4 September 2007

(C) Springer-Verlag 2007

\begin{abstract}
We propose an exact branch-and-bound algorithm for the problem of maximizing the minimum machine completion time on identical parallel machines. The proposed algorithm is based on tight lower and upper bounds as well as an effective symmetry-breaking branching strategy. Computational results performed on a large set of randomly generated instances attest to the efficacy of the proposed algorithm.
\end{abstract}

Keywords Scheduling $\cdot$ Identical parallel machines $\cdot$ Branch-and-bound

MSC classification 90B35

\section{Introduction}

We investigate the problem of maximizing the minimum machine completion time on identical parallel machines. This problem has been first described by Deuermeyer et al. (1982) and is formally described as follows: a set $J$ of $n$ jobs has to be scheduled on $m$ identical parallel machines (with $n>m \geq 2$ ). Each job $j \in J$ has to be processed non-preemptively for $p_{j}$ units of time. The processing times are assumed to be deterministic and integers. Each machine processes at most one job at one time and each job cannot be processed by more than one machine at one time. The machines process jobs continuously from time zero onwards and all jobs are ready for

\footnotetext{
M. Haouari $(\varangle) \cdot$ M. Jemmali

Combinatorial Optimization Research Group-ROI, Ecole Polytechnique de Tunisie,

BP 743, 2078 La Marsa, Tunisia

e-mail: mohamed.haouari@ept.rnu.tn

M. Haouari

Faculty of Business Administration, Bilkent University, Ankara, Turkey
} 
processing at time zero (thus, a machine never needs to wait idle for a job becoming available). Let $J_{i} \subset J$ denote the set of jobs assigned to machine $M_{i}(i=1, \ldots, m)$, then the completion time of $M_{i}$ is defined as $C_{i}=\sum_{j \in J_{i}} p_{j}$. The problem is to find a schedule that maximizes the minimum machine completion time $C_{\min }=\min _{1 \leq i \leq m} C_{i}$. Using the standard three-field notation of Lawler et al. (1993), this problem is denoted $P \| C_{\text {min }}$.

An application of $P \| C_{\min }$ arises in the context of regional allocation of investments as follows. Given a set of $n$ investment projects to be carried out in $m$ different regions. Each project $j(j=1, \ldots, n)$ is expected to generate a revenue $p_{j}$ (or alternatively, $p_{j}$ might represent the number of generated new jobs) and will be entirely carried out in a chosen region $i(i=1, \ldots, m)$. For the sake of equity, the projects should be allocated to the different regions so as to maximize the minimal total regional revenue (or number of generated new jobs). Another application arises in the process of scheduling the replacement parts for the repeated repair of a machine. This problem occurs for instance in the context of modular gas turbine aircraft engines maintenance and is described as follows. A machine includes $m$ identical working parts which frequently require replacement. A finite inventory of $n$ spares is initially available. In most cases the inventory consists of both new as well as refurbished parts. Hence, these spares may have different field lives. Let $p_{j}(j=1, \ldots, n)$ denotes the (deterministic) field life of spare $j$. The problem is to find a replacement part sequencing that maximizes the total time elapsed before the inventory is eventually replenished.

To the best of our knowledge, the literature related to $P \| C_{\min }$ is solely devoted to the investigation of approximation algorithms. Deuermeyer et al. (1982) show that the longest processing time (LPT) heuristic has a performance guarantee of $3 / 4$. This analysis has been tightened by Csirik et al. (1992) who show that the exact worst-case ratio of LPT is $(3 m-1) /(4 m-2)$. Finally, Woeginger (1997) gave a polynomial-time approximation scheme for this problem. Moreover, the on-line version of the problem has been addressed by Azar and Epstein (1997) and Tan and He (2002). Actually, this scanty literature is in sharp contrast to the huge number of papers that have been devoted so far to the investigation of the "dual" of $P \| C_{\min }$. Indeed, if the objective is to minimize $\left\{\max _{1 \leq i \leq m} C_{i}\right\}$ instead of maximizing $\left\{\min _{1 \leq i \leq m} C_{i}\right\}$, then the problem turns out to be the well-known $P \| C_{\max }$. This latter problem is definitely the most classical $\mathcal{N} \mathcal{P}$-hard parallel machine scheduling problem. At this point, it is worth noting that while these two problems are in general different, it is easy to see that for the special two-machine case they are strictly equivalent. Hence, $P \| C_{\min }$ is $\mathcal{N} \mathcal{P}$-hard.

The objective of this paper is to present an exact branch-and-bound algorithm for $P \| C_{\min }$. The proposed algorithm is based on tight lower and upper bounds as well as an effective branching scheme which make it feasible to solve to optimality many hard $P \| C_{\min }$ instances. The remainder of the paper is organized as follows. In Sect. 2, we describe several upper bounds. In Sect. 3, we describe an optimization-based heuristic. In Sect. 4, we describe the branching scheme as well several important features of the branch-and-bound algorithm. In Sect. 5, we present the results of extensive computational experiments. Finally, some concluding remarks are provided.

In the sequel, w.l.o.g it is assumed that $p_{1} \geq p_{2} \geq \cdots \geq p_{n}$ and $C_{1} \leq C_{2} \leq \cdots \leq$ $C_{m}$. 


\section{Upper bounds}

It is well-known that the global effectiveness of a branch-and-bound algorithm strongly relies on the tightness of the embedded upper bounding procedure (for a maximization problem). In this section, we propose several upper bounds for $P \| C_{\min }$.

\subsection{A simple upper bound}

Obviously, we have

$$
\sum_{i=1}^{m} C_{i} \geq m C_{1}
$$

Since, $\sum_{i=1}^{m} C_{i}=\sum_{j=1}^{n} p_{j}$, thus we get a simple $O(n)$ upper bound

$$
U_{0}=\left\lfloor\frac{\sum_{j=1}^{n} p_{j}}{m}\right\rfloor
$$

where $\lfloor a\rfloor$ represents the largest integer that is smaller than or equal to $a$.

\subsection{Upper bounds derived from the $P \| C_{\max }$}

Now, we elucidate the close relationship between $P \| C_{\min }$ and $P \| C_{\max }$ and we show that lower bounds of the latter can be used to derive upper bounds for the former.

Lemma 2.1 Given an instance I of $P \| C_{\min }$ and a valid lower bound $L(I)$ on the optimal make span of the corresponding $P \| C_{\max }$, then a valid upper bound on the optimal solution of the $P \| C_{\min }$ instance is

$$
U(I)=\left\lfloor\frac{\sum_{j=1}^{n} p_{j}-L(I)}{m-1}\right\rfloor
$$

Proof It suffices to observe that $L(I) \leq C_{m} \Rightarrow \sum_{i=1}^{m-1} C_{i} \leq \sum_{j=1}^{n} p_{j}-L(I)$. Moreover, $(m-1) C_{1} \leq \sum_{i=1}^{m-1} C_{i}$. Thus, we get $C_{1} \leq \frac{\sum_{j=1}^{n} p_{j}-L(I)}{m-1}$.

Consequently, any valid lower bound for $P \| C_{\max }$ having a complexity $O(f(n))$ might be used to derive a corresponding upper bound for $P \| C_{\min }$ having a complexity $O(\max (n, f(n))$ (in practice, both bounds would have the same complexity). The $P \| C_{\max }$ is a fundamental scheduling problem that has been extensively investigated, and several lower bounds have been proposed in the literature. For instance, Dell' Amico and Martello (1995) have proposed the following simple linear-time lower 
bound

$$
L_{1}=\max \left(p_{1}, p_{m}+p_{m+1},\left\lceil\sum_{j=1}^{n} p_{j} / m\right\rceil\right)
$$

(recall that the jobs are indexed so that $p_{1} \geq p_{2} \geq \cdots \geq p_{n}$ ). Moreover, Dell' Amico and Martello (1995) have developed a tricky $O\left(n^{2}\right)$-time lower bound $L_{D M}$ which is based on the equivalence between the decision problems corresponding to the bin packing problem (BPP) and $P \| C_{\max }$, respectively. Indeed, checking whether $n$ jobs could be processed on the $m$ machines such that the makespan does not exceed a trial value $C$ is equivalent to checking whether $n$ items can be packed into $m$ bins where the capacity of each bin is equal to $C$. A "no" answer to $P \| C_{\max }$ decision problem is provided if a lower bound on the minimal number of bins (machines) that are required for packing the $n$ items (jobs) exceeds $m$. Consequently, a valid lower bound for $P \|$ $C_{\max }$ is $C^{*}+1$ where $C^{*}$ is the largest value of $C$ that yields a "no" answer. The BPP lower bound that has been proposed by Dell' Amico and Martello (1995) can be briefly described as follows: for each integer $p \in\left\{p_{m+2}, p_{m+3}, \ldots, p_{n}\right\}$ and $p \leq \frac{C}{2}$, let

$$
\begin{aligned}
& J_{1}=\left\{j \in J ; C-p<p_{j}\right\} \\
& J_{2}=\left\{j \in J ; \frac{C}{2}<p_{j} \leq C-p\right\} \\
& J_{3}=\left\{j \in J ; p \leq p_{j} \leq \frac{C}{2}\right\}
\end{aligned}
$$

and define

$$
\begin{aligned}
& B P P_{1}(C, p)=\left|J_{1}\right|+\left|J_{2}\right|+\max \left(0,\left[\frac{\sum_{j \in J_{3}} p_{j}-C\left|J_{2}\right|+\sum_{j \in J_{2}} p_{j}}{C}\right]\right) \\
& B P P_{2}(C, p)=\left|J_{1}\right|+\left|J_{2}\right|+\max \left(0,\left[\frac{\left|J_{3}\right|-\sum_{j \in J_{2}}\left\lfloor\frac{C-p_{j}}{p}\right\rfloor}{\left\lfloor\frac{C}{p}\right\rfloor}\right]\right)
\end{aligned}
$$

Thus, a valid BPP lower bound is $L B_{B P P}=\max _{p}\left\{\max \left(B P P_{1}(C, p), B P P_{2}(C, p)\right)\right\}$. Hence, the corresponding $P \| C_{\max }$ bound is denoted by $L_{D M}$.

Haouari et al. (2006) observe that a practical way for (possibly) improving a lower bound is to consider subset of machines and/or jobs. This observation is based on the following lemma.

Lemma 2.2 (Haouari and Gharbi 2004) In any feasible schedule of a parallel machines problem with $n$ jobs and $m$ machines, there is at least a set of $k$ machines $(1 \leq k \leq m)$ which must process at least $\lambda_{k}=k\lfloor n / m\rfloor+\min (k, \rho)$ jobs, where $\rho=n-m\lfloor n / m\rfloor$. 
Based on this lemma, Haouari et al. (2006) have derived from $L_{1}$ and $L_{D M}$ two stronger $P \| C_{\max }$ lower bounds to which we refer as $L_{2}$ and $L_{3}$, respectively. The complexity of these two bounds are $O\left(n^{2}\right)$ and $O\left(n^{3}\right)$, respectively. In the sequel, we denote by $U_{i}(i=1,2,3)$ to the upper bounds that are derived from $L_{i}$, respectively.

Example 2.3 Consider the instance with $n=5, m=3, p_{1}=118, p_{2}=107$, $p_{3}=86, p_{4}=81, p_{5}=80$. We have $U_{0}=\left\lfloor\frac{472}{3}\right\rfloor=157$, and $L_{1}=\max (118,86+$ $\left.81,\left\lceil\frac{472}{3}\right\rceil\right)=167$. Thus, $U_{1}=\left\lfloor\frac{472-167}{3-1}\right\rfloor=152$.

\subsection{Enhancement procedures}

In order to get tighter upper bounds, we propose two enhancement procedures which aim at strengthening previously developed upper bounds.

\subsubsection{A lifting procedure for $P \| C_{\min }$}

An interesting consequence of Lemma 2.2 is the following corollary.

Corollary 2.4 In any feasible schedule of a parallel machines problem with $n$ jobs and $m$ machines, there is at most a set of $k$ machines $(1 \leq k \leq m)$ which must process at most $\mu_{k}=k\lfloor n / m\rfloor+\max (0, \rho-m+k)$ jobs.

Proof Obviously, for $k=m$ we have $\mu_{k}=n$. For $1 \leq k \leq m-1$, we have $\mu_{k}=n-\lambda_{m-k}$. Thus, we get $\mu_{k}=\left\{\begin{array}{l}k\lfloor n / m\rfloor, \text { for } 1 \leq k \leq m-\rho \\ k\lfloor n / m\rfloor+\rho-m+k, \text { for } m-\rho<k \leq m .\end{array}\right.$

Hence, there are at most $\mu_{k}$ jobs that are scheduled on any subset of $k$ machines $(k=1, \ldots, m)$. Consequently, if we consider the auxiliary $P \| C_{\min }$ instance $I_{k}$ that is defined on $k$ machines and the job-set $J_{k}=\left\{1, \ldots, \mu_{k}\right\}$ (i.e., $J_{k}$ includes the $\mu_{k}$ jobs that have the largest processing times), then its minimum completion time $C_{\min }\left(I_{k}\right)$ is a valid upper bound on the minimum completion time of the $P \| C_{\min }$ instance. Therefore, if $U\left(I_{k}\right)$ denotes an upper bound on $C_{\min }\left(I_{k}\right)$, then a valid lifted upper bound for the $P \| C_{\min }$ instance is

$$
\bar{U}=\min _{1 \leq k \leq m} U\left(I_{k}\right)
$$

Consequently, from each upper bound $U_{i}(i=0, \ldots, 3)$ we can use (2.5) to derive a corresponding lifted upper bound $\bar{U}_{i}$. Clearly, if the computation of $U$ requires $O(f(n))$ time, then $\bar{U}$ requires the computation of $m$ bounds and can therefore be computed in $O(m f(n))$ time.

Example 2.5 Continued. We have $\rho=2$. Thus, for $k=1$ we get $\mu_{1}=1$, $J_{1}=\{1\}$ and $U_{0}\left(I_{1}\right)=\left\lfloor\frac{118}{1}\right\rfloor=118$. For $k=2$, we get $\mu_{2}=3, J_{2}=\{1,2,3\}$ and $U_{0}\left(I_{2}\right)=\left\lfloor\frac{118+107+86}{2}\right\rfloor=155$. For $k=3$, we get $\mu_{3}=5, J_{3}=\{1,2,3,4,5\}$ and $U_{0}\left(I_{3}\right)=\left\lfloor\frac{118+107+86+81+80}{3}\right\rfloor=157$. Thus we get $\bar{U}_{0}=118$. Note that in this case we have $\bar{U}_{0}<U_{1}<U_{0}$. 


\subsubsection{Second enhancement procedure}

This procedure is based on the following observation. Given an upper bound $U$, we can derive a possibly better valid upper bound $\hat{U}$ by solving the following subset sum problem (SSP).

For each job $j(j=1, \ldots, n)$, denote by $x_{j}$ the binary variable that takes the value 1 if job $j$ is assigned to the machine having the minimum completion time and 0 otherwise. Thus, a valid upper bound is

$$
\hat{U}=\operatorname{Max} \sum_{j \in J} p_{j} x_{j}
$$

subject to:

$$
\begin{aligned}
& \sum_{j \in J} p_{j} x_{j} \leq U, \\
& x_{j} \in\{0,1\}, \quad \forall j \in J .
\end{aligned}
$$

It is well-known that although being an $\mathcal{N} \mathcal{P}$-hard problem, the SSP can be efficiently solved in a pseudo-polynomial time (see, Pisinger 2003). In the sequel, we denote by $\hat{U}_{i}(i=0, \ldots, 3)$ the bounds that are obtained through enhancing the bounds $U_{i}$ via solving an SSP, respectively.

\subsubsection{A hybrid enhancement procedure}

We have implemented a hybrid enhancement procedure which is based on a combination of both above-described enhancements procedures. More precisely, for each upper bound $U_{i}(i=0, \ldots, 3)$, we derive

$$
U_{i}^{*}=\min _{1 \leq k \leq m} \hat{U}_{i}\left(I_{k}\right)
$$

Hence, instead of using an upper bound $U_{i}\left(I_{k}\right)$ for instance $I_{k}$ (as in 2.5), we solve an SSP for each $k$, and then we use the enhanced resulting value $\hat{U}_{i}\left(I_{k}\right)$.

Furthermore, for each bound $U_{i}(i=1,2,3)$ which is based on a $P \| C_{\max }$ lower bound $L_{i}$, we have used in (3) the tightened value $\hat{L}_{i}$ which is given by

$$
\hat{L}_{i}=\operatorname{Min} \sum_{j \in J} p_{j} y_{j}
$$

subject to:

$$
\begin{aligned}
& \sum_{j \in J} p_{j} y_{j} \geq L_{i}, \\
& x_{j} \in\{0,1\}, \quad \forall j \in J .
\end{aligned}
$$


where the binary variable $y_{j}$ is defined for each job $j(j=1, \ldots, n)$ and takes the value 1 if job $j$ is assigned to the machine having the maximum completion time and 0 otherwise. This hybrid enhancement strategy has been assessed through computational experiments on randomly generated instances. For these instances, the enhanced upper bounds exhibit an excellent performance. Indeed, in $92.32 \%$ of the cases $U_{3}^{*}$ yields a proven optimal value.

\section{A heuristic algorithm}

Now, we turn our attention to the description of an effective heuristic which constitutes the second key feature of our branch-and-bound algorithm. This heuristic is in the spirit of the approach that has been implemented by Haouari et al. (2006) for $P \| C_{\max }$. Basically, the proposed heuristic is a multi-start local search method which requires iteratively solving a sequence of $P 2 \| C_{\min }$ instances. At each iteration, a pair of machines $M_{1}$ and $M_{m}$ are selected (recall that $C_{1} \leq C_{2} \leq \cdots \leq C_{m}$ ) and the resulting $P 2 \| C_{\min }$ instance which is defined on the jobset $\tilde{J}=J_{1} \cup J_{m}$ is solved to optimality. To that aim, the $P 2 \| C_{\min }$ is reformulated as the following subset sum problem

$$
\operatorname{Maximize} \sum_{j \in \tilde{J}} p_{j} z_{j}
$$

subject to:

$$
\begin{aligned}
& \sum_{j \in \tilde{J}} p_{j} z_{j} \leq\left\lfloor\sum_{j \in \tilde{J}} p_{j} / 2\right\rfloor \\
& x_{j} \in\{0,1\}, \quad \forall j \in \tilde{J},
\end{aligned}
$$

where the binary variable $z_{j}$ is defined for each job $j \in \tilde{J}$ and takes the value 1 if job $j$ is assigned to the machine having the minimum completion time and 0 otherwise.

Let $C_{\min }^{m}$ denote the minimum completion time that is obtained after solving the corresponding subset sum problem. If an improved two-machine schedule has been obtained (hence, $C_{\min }^{m}>C_{1}$ ), then the schedules of machines $M_{1}$ and $M_{m}$ are replaced by the new ones, the new values $C_{k}(k=1, \ldots, m)$ are computed, the machines are reindexed so that $C_{1} \leq C_{2} \leq \cdots \leq C_{m}$, and the procedure is reiterated (i.e., machines $M_{1}$ and $M_{m}$ are rescheduled). Otherwise, the pair of machines $\left(M_{1}, M_{m-1}\right)$ is selected and the corresponding $P 2 \| C_{\text {min }}$ instance is solved as a subset sum problem and so on. The procedure is stopped if no improvement has been achieved after sequentially considering all of the machine pairs $\left(M_{1}, M_{k}\right)(k=m, m-1, \ldots, 2)$. A pseudo-code of the heuristic is described below.

Step 0. (Initialization)

Generate an initial schedule $\sigma$.

Step 1. (Computation of the completion times)

Compute $C_{k}$ for $k=1, \ldots, m$, Set $k=m$. 


\section{Step 2. (Rescheduling of a machine pair)}

Solve using (13)-(15) the $P 2 \| C_{\text {min }}$ instance that is defined on $M_{1}$ and $M_{k}$.

Let $C_{\min }^{k}$ denotes the obtained minimum completion time.

Step 3. (Solution update)

If $C_{\min }^{k}<C_{1}$ then update $\sigma$ and go to Step 1 .

Step 4. (Termination test)

If $(k>2)$ then Set $k=k-1$ and go to Step 2, otherwise Stop.

In order to produce an improved solution, the above described heuristic is reiterated iter times (where the parameter iter is set empirically) after varying the initial schedule. The starting solutions are obtained with a randomized LPT rule. This procedure iteratively selects two unscheduled jobs with the longest processing times and then randomly assigns one out of this pair to the first available machine. In our implementation, we set the maximal number of starting solutions (iter) to 100 . However, the generation process is prematurely halted if a proven optimal solution (i.e., equal to an upper bound) is generated.

\section{A branch-and-bound algorithm}

\subsection{Solution representation}

Let $S$ denote a feasible $P \| C_{\min }$ solution with a corresponding job partition $J_{1}, J_{2}, \ldots, J_{m}$. Note that since the problem exhibits a natural symmetry inherent to identical machines and (possibly) some indistinguishable jobs (i.e., having identical processing times), we can associate with $S$ a set of alternative symmetric solutions that might be generated by re-indexing machines and/or identical jobs. Actually, the number of such alternative symmetric solutions is generally huge. For instance, even for the apparently "benign" case where all jobs are different, we can associate with any feasible solution $S$, a set of $m !-1$ alternative symmetric solutions. Obviously, this symmetry might significantly increase the computational burden and challenge the efficacy of an optimization algorithm. In order to avoid this serious drawback, we propose an efficient symmetry-breaking representation. It is noteworthy that the concept of symmetry-breaking (or -defeating) is widely used in computational integer programming for improving discrete model representations (see, Sherali and Smith 2001). We denote by $n_{i}(i=1, \ldots, m)$ the number of jobs that are assigned to machine $M_{i}$ (i.e., $\left.n_{i}=\left|J_{i}\right|\right)$. Each subset $J_{i}(i=1, \ldots, m)$ is represented by a permutation $\sigma_{i}=\left(\sigma_{i}^{1}, \sigma_{i}^{2}, \ldots, \sigma_{i}^{n_{i}}\right)$ of the $n_{i}$ job indices. Hence, we associate to each solution $S$ a permutation $\sigma(S)=\sigma_{1} \sigma_{2} \ldots \sigma_{m}$. Such a permutation is said to be valid if it satisfies the following conditions:

(C1) $\sigma_{i}^{k}<\sigma_{i}^{k+1}$, for $k=1, \ldots, n_{i}-1, i=1, \ldots, m$

(C2) $\sum_{k=1}^{n_{i}} p_{\sigma_{i}^{k}} \leq \sum_{k=1}^{n_{i+1}} p_{\sigma_{i+1}^{k}}$, for $i=1, \ldots, m-1$

(C3) if for some $i(i=1, \ldots, m-1)$, we have $\sum_{k=1}^{n_{i}} p_{\sigma_{i}^{k}}=\sum_{k=1}^{n_{i+1}} p_{\sigma_{i+1}^{k}}$ then $\sigma_{i}^{1}<\sigma_{i+1}^{1}$ 
(C4) if for some $j(j=1, \ldots, m-1)$, we have $p_{j}=p_{j+1}$ then job $j+1$ is sequenced after job $j$ in $\sigma$.

Condition (C1) specifies that each subsequence $\sigma_{i}(i=1, \ldots, m)$ is a nondecreasing list of the job indices. Condition (C2) requires that the machines are indexed according to nondecreasing completion times. Condition (C3) specifies that ties are broken in favor of the machine having the smallest job index. Condition $(\mathrm{C} 4)$ requires that if two jobs $j$ and $j+1$ are identical and $j$ is assigned to a machine $M_{\alpha}$ then $j+1$ is necessarily assigned to machine $M_{\beta}$ such that $\beta \geq \alpha$.

The main advantage of this representation is that each set of alternative symmetric solutions is represented by a unique permutation of the $n$ jobs.

Example 4.1 Consider the instance with $n=8, m=3, p_{1}=15, p_{2}=12, p_{3}=12$, $p_{4}=10, p_{5}=9, p_{6}=8, p_{7}=5, p_{8}=5$. Let $S$ denote the solution which is specified by the following assignment: jobs 1, 6, and 7 are assigned to a first machine, jobs 2, 5, and 8 are assigned to a second machine, and jobs 3 and 4 are assigned to a third machine. The only valid representation of this solution is $\sigma(S)=\sigma_{1} \sigma_{2} \sigma_{3}$ where $\sigma_{1}=(2,4), \sigma_{2}=(3,5,7)$, and $\sigma_{3}=(1,6,8)$.

Conversely, given a valid permutation $\sigma^{*}$ and a (feasible) threshold value $C_{\min }$, one can construct in $O(n)$ time a feasible solution $S$ having a minimum completion time equal to $C_{\min }$ and satisfying $\sigma(S)=\sigma^{*}$. Clearly, the jobs that are assigned to $M_{1}$ are $\left\{\sigma^{*}(1), \sigma^{*}(2), \ldots, \sigma^{*}\left(v_{1}\right)\right\}$ where $v_{1}$ is the smallest integer $v$ satisfying $\sum_{j=1}^{v} p_{\sigma^{*}(j)} \geq C_{\min }$. Next, the jobs that are assigned to $M_{k}(2 \leq k \leq m-1)$ are $\left\{\sigma^{*}\left(v_{k-1}+1\right), \ldots, \sigma^{*}\left(v_{k}\right)\right\}$ where $v_{k}$ is the smallest integer $v$ satisfying $\sum_{j=v_{k-1}+1}^{v} p_{\sigma^{*}(j)} \geq C_{k-1}$. Finally, the unscheduled jobs $\left\{\sigma^{*}\left(v_{m-1}+1\right), \ldots, \sigma^{*}(n)\right\}$ are assigned to $M_{m}$.

\subsection{Branching scheme and data representation}

Since each solution is represented by a permutation of the $n$ jobs, we have adopted the following branching scheme. Each node $N_{l}$ of level $l$ of the search tree corresponds to a partial valid permutation $\sigma\left(N_{l}\right)=\left(\sigma_{1}, \sigma_{2}, \ldots, \sigma_{l}\right)$ of $l$ jobs. Therefore, the corresponding set of unscheduled jobs is $\bar{J}\left(N_{l}\right)=J \backslash\left\{\sigma_{1}, \sigma_{2}, \ldots, \sigma_{l}\right\}$. Obviously, the root node $N_{0}$ corresponds to the empty permutation. Each child of node $N_{l}$ corresponds to appending an unscheduled job $j_{0} \in \bar{J}\left(N_{l}\right)$ to $\sigma\left(N_{l}\right)$ and thus getting an extended partial permutation $\left(\sigma_{1}, \sigma_{2}, \ldots, \sigma_{l}, j_{0}\right)$. This branching strategy amounts to sequentially loading $M_{1}, M_{2}, \ldots, M_{m}$, in that order, while taking heed of Conditions (C1)-(C4).

With each node $N$ at level $l$ of the search tree is associated the following data:

- $\sigma(N)$ : valid permutation of $l$ jobs

- $J(N)$ : subset of scheduled jobs $(|J(N)|=l)$

- $\quad i(N)$ : index of the last loaded machine

- $\quad L(N)$ : total workload of machine $M_{i(N)}$

- $C_{1}(N)$ : total workload of machine $M_{1}$

- $\quad a(N)$ : lower bound on the workload of machine $M_{i(N)}$

- $\quad b(N)$ : upper bound on the workload of machine $M_{i(N)}$ 
- $\quad j(N)$ : index of the last scheduled job (i.e., $j(N)=\sigma_{l}$ )

- $j_{0}(N)$ : smallest index of the jobs scheduled on $M_{i(N)-1}$

- $j_{1}(N):$ smallest index of the jobs scheduled on $M_{i(N)}$

- $\bar{J}(N)$ : set of unscheduled jobs that are candidate to be scheduled on $M_{i(N)}$.

The data associated with the root node $N_{0}$ is : $\sigma\left(N_{0}\right)=\emptyset, J\left(N_{0}\right)=\emptyset, i\left(N_{0}\right)=1$, $L\left(N_{0}\right)=0, C_{1}\left(N_{0}\right)=0, a\left(N_{0}\right)=L B+1$ (where $L B$ denotes the value of the heuristic solution), $b\left(N_{0}\right)=U B$ (where $U B$ denotes the value of an upper bound which is computed at the root node), $j\left(N_{0}\right)=0, j_{0}\left(N_{0}\right)=0, j_{1}\left(N_{0}\right)=0$, and $\bar{J}\left(N_{0}\right)=J$. Now, we provide some details for a non-root node $N$.

Computation of $a(N)$ : If $i(N)=1$ then $a(N)=L B+1$ (because we are seeking for a solution that strictly outperforms the heuristic one). Moreover, since only valid permutations are generated, then from conditions $(\mathrm{C} 2)$ and $(\mathrm{C} 3)$, we get

$$
a(N):\left\{\begin{array}{l}
C_{i(N)-1}, \text { if } i(N)>1 \text { and } j_{1}(N)>j_{0}(N) \\
C_{i(N)-1}+1, \text { if } i(N)>1 \text { and } j_{1}(N)<j_{0}(N) .
\end{array}\right.
$$

Computation of $b(N)$ : An upper bound on the total workload of $M_{i(N)}$ is computed by considering a reduced $P \| C_{\min }$ instance defined on $m^{\prime}=m-i(N)+1$ machines and $n^{\prime}=|\bar{J}(N)|+1$ jobs. The jobset comprises the unscheduled jobs as well as a dummy job $n+1$ corresponding to the subset of jobs that have been already assigned to $M_{i(N)}$. Thus, the processing time of this dummy job is equal to the total workload of machine $M_{i(N)}$ (i.e., $p_{n+1}=L(N)$ )

Computation of $\bar{J}(N)$ : The set of unscheduled jobs that are candidate to be scheduled on $M_{i(N)}$ immediately after $j(N)$ is

$$
\bar{J}(N)=\left\{j \in J \backslash J(N): j>j(N) \text { and } p_{j}+L(N) \leq b(N)\right\}
$$

Assume that node $N$ is branched and that each child node $N^{+}$is obtained by appending a job $j \in J \backslash J(N)$ to $\sigma(N)$. First, consider the situation where $1<i(N) \leq m-1$. Two cases may occur:

Case (i): $\quad L(N)<a(N)$ and $j \in \bar{J}(N))$ : in this case, additional jobs must be assigned to machine $M_{i(N)}$ and job $j$ is a valid candidate. Therefore, for node $N^{+}$we define $\sigma\left(N^{+}\right)=\sigma(N) j, J\left(N^{+}\right)=J(N) \cup\{j\}, i\left(N^{+}\right)=i(N)$, $L\left(N^{+}\right)=L(N)+p_{j}, C_{1}\left(N^{+}\right)=C_{1}(N), a\left(N^{+}\right)=a(N), j\left(N^{+}\right)=j$, $j_{0}\left(N^{+}\right)=j_{0}(N), j_{1}\left(N^{+}\right)=j_{1}(N)$. Moreover, $b\left(N^{+}\right)$and $\bar{J}\left(N^{+}\right)$are computed directly.

Case (ii): $L(N) \geq a(N)$ or $j \notin \bar{J}(N)$ : in this case, no further job should be assigned to machine $M_{i(N)}$ and job $j$ should be assigned to the next machine $M_{i(N)+1}$. Therefore, the data of $N^{+}$is : $\sigma\left(N^{+}\right)=\sigma(N) j, J\left(N^{+}\right)=$ $J(N) \cup\{j\}, i\left(N^{+}\right)=i(N)+1, L\left(N^{+}\right)=p_{j}, C_{1}\left(N^{+}\right)=C_{1}(N)$, $a\left(N^{+}\right)=a(N)+\delta$ where $\delta=1$ if $j<j_{1}(N)$ and 0 , otherwise, $j\left(N^{+}\right)=$ $j, j_{0}\left(N^{+}\right)=j_{1}(N), j_{1}\left(N^{+}\right)=j$. Here again, $b\left(N^{+}\right)$and $\bar{J}\left(N^{+}\right)$are computed directly.

Now, consider the special case where $i(N)=1$. Here, three cases may occur: 
Case (iii): $\quad L(N)<a(N)$ and $j \in \bar{J}(N)$ : in this case additional jobs must be assigned to machine $M_{1}$ and job $j$ is a valid candidate. We set $C_{1}\left(N^{+}\right)=$ $L(N)+p_{j}$. The remaining data are updated similarly to Case $(i)$.

Case (iv): $\quad L(N) \geq a(N)$ and $j \notin \bar{J}(N)$ : in this case job $j$ cannot be assigned to machine $M_{1}$ and requires to be therefore assigned to machine $M_{2}$. This situation is similar to Case (ii).

Case (v): $\quad L(N) \geq a(N)$ and $j \in \bar{J}(N)$ : in this case, job $j$ is either assigned to machine $M_{1}$ or to machine $M_{2}$. Of course, in this latter case no further job would be assigned to $M_{1}$. It is noteworthy that this yields two different descendent nodes $N_{1}^{+}$and $N_{2}^{+}$having the same partial permutation. Therefore, the data associated with nodes $N_{1}^{+}$and $N_{2}^{+}$are updated similarly to Cases (ii) and (iii), respectively.

\subsection{Implementation details}

Implemented bounds. We have performed extensive computational experiments in order to assess different combinations of upper bounding procedures. We found that an efficient implementation is obtained when $U_{3}^{*}$ is computed at the root node and $\bar{U}_{1}$ at non-root nodes. While the former upper bound is often very tight, but is (relatively) time-consuming, the latter one performs reasonably well and is very fast. Moreover, the lower bound at the root node $(L B)$ is delivered by the heuristic which is described in Sect. 3.

Node pruning. Obviously, whenever a solution having a minimum completion time satisfying $C_{\min }>L B$ is found, the incumbent value is updated and all the active nodes $N$ having $C_{1}(N) \leq L B$ are pruned. In addition, a node $N$ is pruned if any of the following conditions holds:

$-\quad b(N)<a(N)$

$-\quad i(N)=m-1$ and $L(N) \geq a(N)$.

The first condition refers to the case where $N$ is infeasible (i.e., the corresponding permutation is not valid). The second condition refers to the case where $N$ is a leaf of the search tree. Indeed, if $m-1$ machines are loaded, then the unscheduled jobs are necessarily assigned to the remaining unloaded machine $M_{m}$. In this case, the incumbent value is updated by setting $L B=\min \left(L B, C_{1}(N), \sum_{j \in \bar{J}(N)} p_{j}\right)$.

Taking heed of Condition (C4). Assume that for some node $N$, we have $\{j, j+1$, $\ldots, j+h\} \subset(J \backslash J(N))$ and $p_{j}=p_{j+1}=\cdots=p_{j+h}$. Then, in order to generate a permutation for which Condition (C4) holds, the children nodes that are obtained by appending to $\sigma(N)$ the $h$ jobs $j+1, \ldots, j+h$, respectively, are not created.

Search strategy. We have adopted a depth-first search strategy. In the sequel, we refer to the resulting branch-and-bound algorithm by $B B 1$. 


\subsection{An alternative branching strategy}

In order to get a better insight of the actual pertinence of the proposed symmetrybreaking branching strategy, we have implemented a second version of the proposed depth-first branch-and-bound algorithm with the same upper and lower bounds but with a different branching strategy. This latter strategy is similar to the one that has been previously implemented by Dell'Amico and Martello (1995) for solving $P \| C_{\max }$. Hereafter, we refer to this second version by $B B 2$. On the contrary to the above described branching strategy, the $m$ machines are loaded simultaneously. Indeed, while the root node represents the empty schedule, at level $k(k \geq 1)$ of the search tree, a node represents a partial schedule on the $m$ machines of the job subset $\{1,2, \ldots, k\}$. Given a node at level $k, m$ child nodes are created by assigning job $k+1$ to machines $M_{1}$, $M_{2}, \ldots, M_{m}$, respectively. An associated upper bound is computed by considering a $P m \| C_{\min }$ instance defined on the subset $\bar{J}$ of unscheduled jobs as well as $m$ dummy jobs having processing times $C_{1}, C_{2}, \ldots, C_{m}$, respectively.

Moreover, we have implemented the three following dominance rules:

Rule 1: If for some machine $M_{i}(i=1, \ldots, m-1)$ we have $C_{i}=C_{i+1}$ then job $k+1$ is only assigned to machine $M_{i}$ (otherwise unnecessary equivalent solutions would be created)

Rule 2: If for some job $j(j=1, \ldots, n-1)$ we have $p_{j}=p_{j+1}$ and job $j$ is assigned to machine $M_{i}(i \geq 2)$ then job $j+1$ could not be assigned to any of machines $M_{1}, M_{2}, \ldots, M_{i-1}$ (here again, because unnecessary equivalent solutions would be created)

Rule 3: If $|\bar{J}|<m$ then job $j+1$ could only be assigned to machines $M_{1}, M_{2}, \ldots$, $M_{|\bar{J}|}$ (otherwise, dominated nodes would be created).

\section{Computational results}

We have coded the proposed upper and lower bounds as well as the branch-and-bound algorithms $B B 1$ and $B B 2$ in Microsoft Visual $C++($ Version 6.0). All our experiments were obtained on a Pentium IV 3.2 GHz Personal Computer with 3 GB RAM. We have considered five problem classes that have been randomly generated as described in Dell' Amico and Martello (1995) who used them for testing an exact branch-and-bound algorithm for $P \| C_{\max }$. The processing times were generated according to the following distributions:

- Class 1: discrete uniform distribution on [1,100]

- Class 2: discrete uniform distribution on [20,100]

- Class 3: discrete uniform distribution on [50,100]

- Class 4: normal distribution with Mean 100 and SD 50

- Class 5: normal distribution with Mean 100 and SD 20. 
The number of jobs is ranging between 10 and 10,000, and the number of machines is ranging between 2 and 15 . For each class, and each $(n, m)$ combination, 10 instances were generated. Hence, a total number of 2,050 instances have been generated.

Table 1 displays a summary of the results that were obtained for the upper bounding procedures. In this table, each column corresponds to an $(n, m)$ combination. For each upper bound, we provide the number of times (out of 50) it yields the minimal value over all of the bounds.

We see from this table, that the enhanced bounds exhibit a very good performance. In particular, the bound $U_{3}^{*}$ consistently dominates all the other ones.

In Table 2, we report a summary of the results obtained with $B B 1$. From this table, we see that the implemented bounds are very tight, since for most instances branching was unnecessary. Actually, we found that branching was required for only 166 instances out of 2,050. Moreover, we observe that only 6 instances remained unsolved after reaching the time limit of $800 \mathrm{~s}$.

Table 3 displays the results that were obtained with $B B 2$. Since we have implemented the same upper and lower bounds as in $B B 1$, then here again branching was required for 166 instances out of 2,050. However, we observe that 89 instances remained unsolved after reaching the CPU time limit. This result provides evidence of the superiority of the symmetry-breaking branching strategy that has been implemented in $B B 1$.

In order to get a better picture of the performance of $B B 1$, we have generated an additional class of 260 instances where for each instance with $n$ jobs $(n=10$, $25,50,100,250,500,1,000)$ the processing times are drawn from discrete uniform distribution on $[1, n]$. For these instances, it is expected that only a few jobs have equal processing times. The results are summarized in Table 4 . We see that while all of these instances were optimally solved by $B B 1$ (mostly at the root node), there are seven instances that remained unsolved by $B B 2$.

Finally, we have run our algorithms on a class of perfect packing instances. This class comprises 1,520 instances that have been randomly generated as indicated in Dell' Amico and Martello (1995). A nice property peculiar to this latter class is that the optimal schedule has an equal completion time on each machine (note that in this case $P \| C_{\min }$ and $P \| C_{\max }$ are strictly equivalent). The results are consistent with those obtained for the other problem classes. Indeed, all of these instances were solved to optimality by $B B 1$ and branching was required for only 18 instances. Among these 18 instances, $B B 2$ failed to solve 15 .

\section{Conclusion}

In this paper, we have proposed an exact branch-and-bound algorithm for $P \| C_{\min }$. The proposed algorithm includes several distinctive algorithmic features. In particular, it is based on tight lower and upper bounds as well as an effective symmetry-breaking branching strategy. Computational results attest to the efficacy of the proposed algorithm, which has been able to solve to optimality all but 6 instances out of 3,830 randomly generated instances. 


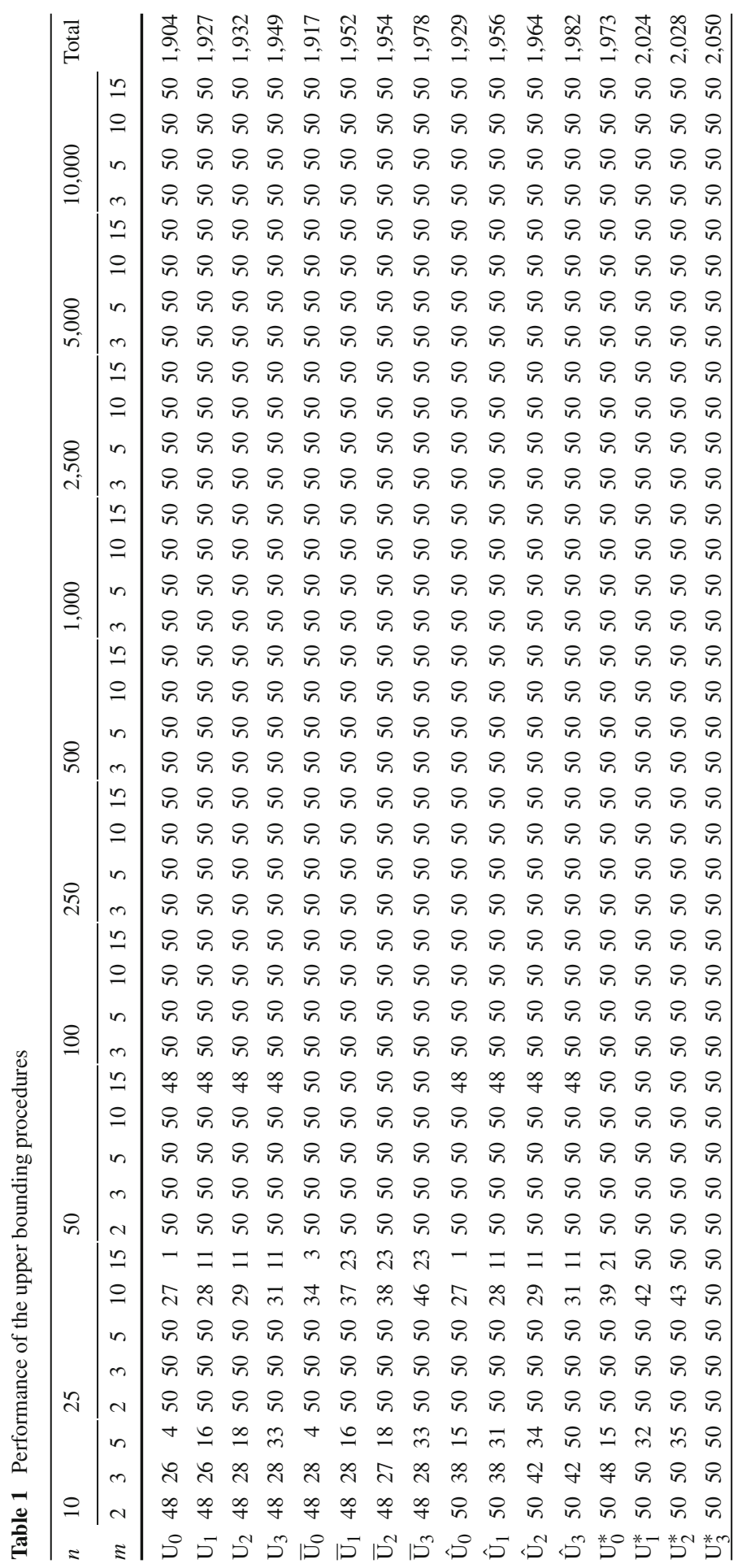


Table 2 Performance of the branch and bound algorithm $B B 1$

\begin{tabular}{|c|c|c|c|c|c|c|c|c|c|c|c|}
\hline \multirow[t]{2}{*}{$n$} & \multirow[t]{2}{*}{$m$} & \multicolumn{2}{|l|}{ Class 1} & \multicolumn{2}{|l|}{ Class 2} & \multicolumn{2}{|l|}{ Class 3} & \multicolumn{2}{|l|}{ Class 4} & \multicolumn{2}{|l|}{ Class 5} \\
\hline & & $N N$ & Time & $N N$ & Time & $N N$ & Time & $N N$ & Time & $N N$ & Time \\
\hline \multirow[t]{3}{*}{10} & 2 & 1 & - & 1 & - & 1 & - & 1 & - & 1 & - \\
\hline & 3 & 90 & 0.001 & 137 & 0.001 & 48 & 0.002 & 215 & 0.002 & 100 & 0.002 \\
\hline & 5 & 128 & 0.002 & 152 & 0.002 & 188 & - & 150 & 0.003 & 140 & 0.002 \\
\hline \multirow[t]{5}{*}{25} & 2 & 1 & - & 1 & - & 1 & 0.002 & 1 & - & 1 & - \\
\hline & 3 & 1 & - & 1 & - & 1 & - & 1 & - & 1 & - \\
\hline & 5 & 1 & - & 1 & - & 1 & - & 1 & - & 1 & - \\
\hline & 10 & 19649828 & 85 & 31675367 & $129(9)$ & 235178 & 1.380 & 4294944.9 & 19.625 & 10014760 & 38.675 \\
\hline & 15 & 24572119 & 128 & 268948 & 1.900 & 42 & 0.006 & 14537221 & 77.878 & 38 & 0.005 \\
\hline \multirow[t]{5}{*}{50} & 2 & 1 & - & 1 & - & 1 & - & 1 & - & 1 & - \\
\hline & 3 & 1 & - & 1 & - & 1 & - & 1 & - & 1 & - \\
\hline & 5 & 1 & - & 1 & - & 1 & - & 1 & - & 1 & - \\
\hline & 10 & & - & 1 & - & 1 & - & 1 & - & 1 & - \\
\hline & 15 & & 0.003 & 21 & 0.001 & 1 & $0.001(8)$ & 4498586.8 & 20.335 & 51 & $0.001(8)$ \\
\hline \multirow[t]{4}{*}{100} & 3 & 1 & - & 1 & - & 1 & - & 1 & - & 1 & - \\
\hline & 5 & 1 & - & 1 & - & 1 & - & 1 & - & 1 & - \\
\hline & 10 & & - & 1 & - & 1 & - & 1 & - & 1 & - \\
\hline & 15 & & - & 1 & - & 1 & - & 1 & - & 1 & - \\
\hline \multirow[t]{5}{*}{250} & 3 & 1 & - & 1 & - & 1 & - & 1 & - & 1 & - \\
\hline & 5 & 1 & - & 1 & - & 1 & - & 1 & - & 1 & - \\
\hline & & & - & & - & & - & & - & & - \\
\hline & 10 & & - & 1 & - & 1 & - & 1 & - & 1 & - \\
\hline & 15 & & - & 1 & - & 1 & - & 1 & - & 1 & - \\
\hline \multirow[t]{4}{*}{500} & 3 & 1 & - & 1 & 0.001 & 1 & 0.001 & 1 & - & 1 & 0.001 \\
\hline & 5 & 1 & - & 1 & - & 1 & - & 1 & - & 1 & - \\
\hline & 10 & & - & 1 & - & 1 & - & 1 & - & 1 & 0.001 \\
\hline & 15 & & - & 1 & 0.001 & 1 & 0.002 & 1 & - & 1 & 0.002 \\
\hline \multirow[t]{4}{*}{1,000} & 03 & 1 & - & 1 & 0.004 & 1 & 0.006 & 1 & - & 1 & 0.004 \\
\hline & 5 & 1 & - & 1 & - & 1 & - & 1 & - & 1 & 0.003 \\
\hline & 10 & & - & 1 & - & 1 & - & 1 & - & 1 & 0.004 \\
\hline & 15 & & - & 1 & 0.006 & 1 & 0.008 & 1 & 0.002 & 1 & 0.006 \\
\hline \multirow[t]{6}{*}{2,500} & 03 & 1 & 0.002 & 1 & 0.016 & 1 & 0.021 & 1 & 0.002 & 1 & 0.016 \\
\hline & 5 & 1 & 0.002 & 1 & 0.002 & 1 & 0.002 & 1 & 0.002 & 1 & 0.016 \\
\hline & 10 & & 0.002 & 1 & 0.002 & 1 & 0.002 & 1 & 0.002 & 1 & 0.016 \\
\hline & 15 & & 0.002 & 1 & 0.02 & 1 & 0.021 & 1 & 0.003 & 1 & 0.019 \\
\hline & 3 & 1 & 0.006 & 1 & 0.07 & 1 & 0.084 & 1 & 0.005 & 1 & 0.062 \\
\hline & 5 & 1 & 0.006 & 1 & 0.004 & 1 & 0.005 & 1 & 0.005 & 1 & 0.050 \\
\hline
\end{tabular}


Table 2 continued

\begin{tabular}{|c|c|c|c|c|c|c|c|c|c|c|c|}
\hline \multirow[t]{2}{*}{$n$} & \multirow[t]{2}{*}{$m$} & \multicolumn{2}{|c|}{ Class 1} & \multicolumn{2}{|c|}{ Class 2} & \multicolumn{2}{|c|}{ Class 3} & \multicolumn{2}{|c|}{ Class 4} & \multicolumn{2}{|c|}{ Class 5} \\
\hline & & $N N$ & Time & $N N$ & Time & $N N$ & Time & $N N$ & Time & $N N$ & Time \\
\hline \multirow[t]{2}{*}{5,000} & 10 & 1 & 0.006 & 1 & 0.005 & 1 & 0.005 & 1 & 0.005 & 1 & 0.047 \\
\hline & 15 & 1 & 0.005 & 1 & 0.08 & 1 & 0.098 & 1 & 0.005 & 1 & 0.070 \\
\hline \multirow[t]{4}{*}{10,000} & 3 & 1 & 0.016 & 1 & 0.519 & 1 & 0.520 & 1 & 0.016 & 1 & 0.294 \\
\hline & 5 & 1 & 0.015 & 1 & 0.016 & 1 & 0.016 & 1 & 0.016 & 1 & 0.177 \\
\hline & 10 & 1 & 0.015 & 1 & 0.016 & 1 & 0.016 & 1 & 0.016 & 1 & 0.177 \\
\hline & 15 & 1 & 0.016 & 1 & 1.588 & 1 & 2.605 & 1 & 0.016 & 1 & 0.575 \\
\hline
\end{tabular}

(-) means that the average CPU time is less than $0.001 \mathrm{~s}$

The figures in brackets indicate the number of solved instances if less than 10

Table 3 Performance of the branch and bound algorithm $B$ B2

\begin{tabular}{|c|c|c|c|c|c|c|c|c|c|c|c|}
\hline \multirow[t]{2}{*}{$n$} & \multirow[t]{2}{*}{$m$} & \multicolumn{2}{|l|}{ Class 1} & \multicolumn{2}{|l|}{ Class 2} & \multicolumn{2}{|c|}{ Class3 } & \multicolumn{2}{|l|}{ Class 4} & \multicolumn{2}{|c|}{ Class 5} \\
\hline & & $N N$ & Time & $N N$ & Time & $N N$ & Time & $N N$ & Time & $N N$ & Time \\
\hline \multirow[t]{5}{*}{10} & 2 & 1 & - & 1 & - & 1 & - & 1 & - & 1 & - \\
\hline & 3 & 48 & 0.001 & 117 & 0.001 & 45 & 0.001 & 207 & 0.001 & 97 & 0.002 \\
\hline & 5 & 1,366 & 0.007 & 1,019 & 0.005 & 582 & 0.004 & 2,159 & 0.013 & 645 & 0.004 \\
\hline & 2 & 1 & - & 1 & - & 1 & 0.002 & 1 & - & 1 & - \\
\hline & 3 & 1 & - & 1 & - & 1 & - & 1 & - & 1 & - \\
\hline \multirow[t]{3}{*}{25} & 5 & 1 & - & 1 & - & 1 & - & 1 & - & 1 & - \\
\hline & 10 & $2,9754,513$ & $181.901(7)$ & $4,6047,433$ & (2) & 1 & $0.007(2)$ & $43,02,480$ & $30.422(2)$ & - & (0) \\
\hline & 15 & - & $(0)$ & 1 & (1) & 1 & $0.003(3)$ & (0) & & 1 & $0.003(3)$ \\
\hline \multirow[t]{5}{*}{50} & 2 & 1 & - & 1 & - & 1 & - & 1 & - & 1 & - \\
\hline & 3 & 1 & - & 1 & - & 1 & - & 1 & - & 1 & - \\
\hline & 5 & 1 & - & 1 & - & 1 & - & 1 & - & 1 & - \\
\hline & 10 & 1 & - & 1 & - & 1 & - & 1 & - & 1 & - \\
\hline & 15 & 1 & $-(8)$ & 1 & 0.001 & 1 & $0.002(9)$ & 1 & $0.002(6)$ & 1 & $-(8)$ \\
\hline \multirow[t]{4}{*}{100} & 3 & 1 & - & 1 & - & 1 & - & 1 & - & 1 & - \\
\hline & 5 & 1 & - & 1 & - & 1 & - & 1 & - & 1 & - \\
\hline & 10 & 1 & - & 1 & - & 1 & - & 1 & - & 1 & - \\
\hline & 15 & 1 & - & 1 & - & 1 & - & 1 & - & 1 & - \\
\hline \multirow[t]{4}{*}{250} & 3 & 1 & - & 1 & - & 1 & - & 1 & - & 1 & - \\
\hline & 5 & 1 & - & 1 & - & 1 & - & 1 & - & 1 & - \\
\hline & 10 & 1 & - & 1 & - & 1 & - & 1 & - & 1 & - \\
\hline & 15 & 1 & - & 1 & - & 1 & - & 1 & - & 1 & - \\
\hline \multirow[t]{4}{*}{500} & 3 & 1 & - & 1 & 0.001 & 1 & 0.001 & 1 & - & 1 & 0.001 \\
\hline & 5 & 1 & - & 1 & - & 1 & - & 1 & - & 1 & - \\
\hline & 10 & 1 & - & 1 & - & 1 & - & 1 & - & 1 & 0.001 \\
\hline & 15 & 1 & - & 1 & 0.001 & 1 & 0.002 & 1 & - & 1 & 0.002 \\
\hline \multirow[t]{6}{*}{1,000} & 3 & 1 & - & 1 & 0.004 & 1 & 0.006 & 1 & - & 1 & 0.004 \\
\hline & 5 & 1 & - & 1 & - & 1 & - & 1 & - & 1 & 0.003 \\
\hline & 10 & 1 & - & 1 & - & 1 & - & 1 & - & 1 & 0.004 \\
\hline & 15 & 1 & - & 1 & 0.006 & 1 & 0.008 & 1 & 0.002 & 1 & 0.006 \\
\hline & 3 & 1 & 0.002 & 1 & 0.016 & 1 & 0.021 & 1 & 0.002 & 1 & 0.016 \\
\hline & 5 & 1 & 0.002 & 1 & 0.002 & 1 & 0.002 & 1 & 0.002 & 1 & 0.016 \\
\hline
\end{tabular}


Table 3 continued

\begin{tabular}{|c|c|c|c|c|c|c|c|c|c|c|c|}
\hline \multirow[t]{2}{*}{$n$} & \multirow[t]{2}{*}{$m$} & \multicolumn{2}{|c|}{ Class 1} & \multicolumn{2}{|c|}{ Class 2} & \multicolumn{2}{|c|}{ Class3 } & \multicolumn{2}{|c|}{ Class 4} & \multicolumn{2}{|c|}{ Class 5} \\
\hline & & $N N$ & Time & $N N$ & Time & $N N$ & Time & $N N$ & Time & $N N$ & Time \\
\hline \multirow[t]{2}{*}{2,500} & 10 & 1 & 0.002 & 1 & 0.002 & 1 & 0.002 & 1 & 0.002 & 1 & 0.016 \\
\hline & 15 & 1 & 0.002 & 1 & 0.02 & 1 & 0.021 & 1 & 0.003 & 1 & 0.019 \\
\hline \multirow[t]{4}{*}{5,000} & 3 & 1 & 0.006 & 1 & 0.07 & 1 & 0.084 & 1 & 0.005 & 1 & 0.062 \\
\hline & 5 & 1 & 0.006 & 1 & 0.004 & 1 & 0.005 & 1 & 0.005 & 1 & 0.050 \\
\hline & 10 & 1 & 0.006 & 1 & 0.005 & 1 & 0.005 & 1 & 0.005 & 1 & 0.047 \\
\hline & 15 & 1 & 0.005 & 1 & 0.080 & 1 & 0.098 & 1 & 0.005 & 1 & 0.070 \\
\hline \multirow[t]{4}{*}{10,000} & 3 & 1 & 0.016 & 1 & 0.519 & 1 & 0.520 & 1 & 0.016 & 1 & 0.294 \\
\hline & 5 & 1 & 0.015 & 1 & 0.016 & 1 & 0.016 & 1 & 0.016 & 1 & 0.177 \\
\hline & 10 & 1 & 0.015 & 1 & 0.016 & 1 & 0.016 & 1 & 0.016 & 1 & 0.177 \\
\hline & 15 & 1 & 0.016 & 1 & 1.588 & 1 & 2.605 & 1 & 0.016 & 1 & 0.575 \\
\hline
\end{tabular}

(-) means that the average CPU time is less than $0.001 \mathrm{~s}$

The figures in brackets indicate the number of solved instances if less than 10

Table 4 Performance of $B B 1$ and $B B 2$ on instances with processing times drawn from $[1, n]$

(-) means that the average CPU time is less than $0.001 \mathrm{~s}$.

The figures in brackets indicate the number of solved instances if less than 10

\begin{tabular}{|c|c|c|c|c|c|}
\hline \multirow[t]{2}{*}{$n$} & \multirow[t]{2}{*}{$m$} & \multicolumn{2}{|l|}{$B B 1$} & \multicolumn{2}{|l|}{$B B 2$} \\
\hline & & $N N$ & Time & $N N$ & Time \\
\hline \multirow[t]{2}{*}{10} & 3 & 1 & - & 1 & - \\
\hline & 5 & 101 & - & 66 & - \\
\hline \multirow[t]{4}{*}{25} & 3 & 1 & - & 1 & - \\
\hline & 5 & 1 & - & 1 & - \\
\hline & 10 & 3954509 & 21.165 & 2545768 & 15.335 \\
\hline & 15 & 12301985 & 73.786 & 242984 & $2.549(3)$ \\
\hline \multirow[t]{4}{*}{50} & 3 & 1 & - & 1 & - \\
\hline & 5 & 1 & - & 1 & - \\
\hline & 10 & 1 & - & 1 & - \\
\hline & 15 & 1 & - & 1 & - \\
\hline \multirow[t]{4}{*}{100} & 3 & 1 & - & 1 & - \\
\hline & 5 & 1 & - & 1 & - \\
\hline & 10 & 1 & - & 1 & - \\
\hline & 15 & 1 & - & 1 & - \\
\hline \multirow[t]{4}{*}{250} & 3 & 1 & - & 1 & - \\
\hline & 5 & 1 & - & 1 & - \\
\hline & 10 & 1 & - & 1 & - \\
\hline & 15 & 1 & - & 1 & - \\
\hline \multirow[t]{4}{*}{500} & 3 & 1 & - & 1 & - \\
\hline & 5 & 1 & 0.001 & 1 & 0.001 \\
\hline & 10 & 1 & - & 1 & - \\
\hline & 15 & 1 & 0.001 & 1 & 0.001 \\
\hline \multirow[t]{4}{*}{1,000} & 3 & 1 & 0.002 & 1 & 0.002 \\
\hline & 5 & 1 & 0.003 & 1 & 0.003 \\
\hline & 10 & 1 & 0.004 & 1 & 0.004 \\
\hline & 15 & 1 & 0.005 & 1 & 0.005 \\
\hline
\end{tabular}


We expect that some of the ideas that have been developed in this paper would prove useful for the exact solution of similar parallel machine scheduling problems, but this would require further investigation.

\section{References}

Azar Y, Epstein L (1997) On-line machine covering. Lect Notes Comput Sci 1284:23-36

Csirik J, Kellerer H, Woeginger G (1992) The exact LPT-bound for maximizing the minimum completion time. Oper Res Lett 11:281-287

Dell'Amico M, Martello S (1995) Optimal scheduling of tasks on identical parallel processors. ORSA J Comput 7:191-200

Deuermeyer BL, Friesen DK, Langston MA (1982) Scheduling to maximize the minimum processor finish time in a multiprocessor system. SIAM J Algorithms Discret Methods 3:190-196

Haouari M, Gharbi A (2004) Lower bounds for scheduling on identical parallel machines with heads and tails. Ann Oper Res 129:187-204

Haouari M, Gharbi A, Jemmali M (2006) Tight bounds for the identical parallel machine scheduling problem. Int Trans Oper Res 13:529-548

Lawler EL, Lenstra JK, Rinnooy Kan AHG, Shmoys D (1993) Sequencing and scheduling: algorithms and complexity. In: Graves SS, Rinnooy Kan AHG, Zipkin P (eds) Handbooks in operations research and management science, vol 4, pp 445-522

Pisinger D (2003) Dynamic programming on the word RAM. Algorithmica 35:437-459

Sherali HD, Smith JC (2001) Improving discrete model representations via symmetry considerations. Manage Sci 47:1396-1407

Tan ZY, He Y (2002) Ordinal on-line scheduling for maximizing the minimum machine completion time. J Comb Optim 6:199-206

Woeginger GJ (1997) A polynomial time approximation scheme for maximizing the minimum completion time. Oper Res Lett 20:149-154 